\title{
ANÁLISE DE NUVENS DE PONTOS GERADAS DE FORMA SEMICONTROLADA PARA VERIFICAÇÃO DO CRESCIMENTO DAS PLANTAS DE MILHO A PARTIR DE DADOS OBTIDOS EM DIFERENTES ALTURAS DE VOO
}

\author{
Ricardo Kwiatkowski Silva e Selma Regina Aranha Ribeiro \\ Universidade Estadual de Ponta Grossa - UEPG, Programa de Pós-Graduação em Computação Aplicada
} Paraná, Brasil

\begin{abstract}
RESUMO
O processamento de fotos digitais obtidas por meio de Aeronaves Remotamente Pilotadas (tradução para Remotely Piloted Aircraft - RPA) constitui um meio eficaz para adquirir informações sobre áreas agrícolas. Desta forma, obtêm-se produtos que permitem monitorá-las. Neste trabalho verifica-se a possibilidade de estimar a altura de uma lavoura localizada nas coordenas UTM 596270, 7224070 (Datum SIRGAS 2000-22S), calculando-se a distância euclidiana do eixo Z de uma mesma área definida pelo eixos X e Y entre o Modelo Digital de Terreno (MDT) e nuvens de pontos obtidas sem a utilização de pontos de controle. Assim, nesta pesquisa não utilizou-se Modelo Digital de Elevação (MDE). Para solucionar o problema da baixa acurácia posicional da nuvem de pontos em relação ao MDT controlado, propõe-se que sejam aplicadas translações nas nuvens de pontos. O método possibilitou estimar a altura, conforme o estádio fenológico em que encontrava-se o milho. No entanto, recomenda-se a aplicação da metodologia somente quando não existem pontos de controle relacionados as fotos da cultura.
\end{abstract}

\section{PALAVRAS-CHAVE}

Nuvens de Pontos, Translação, Processamento Aerofotogramétrico Semicontrolado, Milho

\section{INTRODUÇÃO}

As tecnologias baseadas em sensoriamento remoto são formas eficazes de obter-se informações sobre as culturas. Após o imageamento, processam-se as imagens levantadas para que estas sejam uma fonte de informações sobre as lavouras. Por meio do processamento fotogramétrico das imagens gera-se nuvens de pontos e MDT, que permitem a aferição da altura das plantas.

De acordo com Enciso et al (2019), é possível avaliar a altura na cultura do tomate utilizando-se de dados obtidos por meio da RPA. Bending et al. (2014), propuseram medir a altura com o objetivo de estimar a biomassa, subtraindo-se o MDE do MDT. Outro autor relacionado ao tema (Grenzdörffer, 2014), mediu a altura do milho por meio da correlação existente entre observações de campo e um índice de vegetação.

Assim, tem-se a altura como um parâmetro biofísico que permite acompanhar o desenvolvimento da lavoura, pois plantas que se desenvolvem bem, também irão produzir. O monitoramento também possibilita a detecção de problemas em tempo hábil para mitigar perdas.

A principal contribuição do trabalho é fornecer uma alternativa para estimar a altura das plantas quando o equipamento GNSS (Global Navigation Satellite System) não estiver disponível ou não existirem pontos de controle nas fotos, evitando-se assim que um determinado trabalho de campo não possa ser utilizado.

\section{PROCESSAMENTO DE DADOS DA RPA}

Para realizar o trabalho, planos de voo foram elaborados para obtenção das imagens aéreas utilizando-se do software livre DroneDeploy, compatível com a RPA DJI Mavic 2 Pro. Trata-se de um rotor quadricoptero com sensor GNSS e câmara L1D-20c Hasselblad RGB, para coleta de imagens. 
Na data de 01/11/2019, foi executado um plano de voo para o qual foi determinada uma altura de $100 \mathrm{~m}$ e na data de 03/12/2019 dois planos de voo foram realizados (um a $60 \mathrm{~m}$ e outro a $200 \mathrm{~m}$ de altura). Todos foram programados para sobreposição longitudinal de $80 \%$ e lateral de $70 \%$ das imagens.

Para obter-se as nuvens de pontos, utilizou-se o programa Agisoft Metashape (Agisoft, 2017). Pode-se processar as fotos em tamanho original (Ultra High quality -Ultra alta qualidade) ou em níveis decrescentes de processamento e de tamanho da imagem pelo fator 4. Assim, as imagens levantadas na data de 01/11/2019 foram submetidas ao processo Align Photos (Alinhar Fotos) de forma controlada, por meio da opção Accuracy em modo Highest e as imagens obtidas na data de 03/12/2019 foram submetidas ao processo de alinhamento de forma semicontrolada com diferentes parâmetros de acuracidade: Highest, High, Medium e Low. O processamento de dados segue o fluxo de trabalho, conforme demonstra-se na Figura 1.

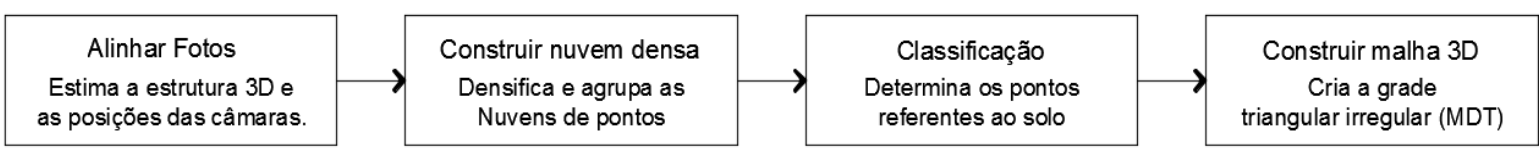

Figura 1. Fluxo de trabalho conforme o programa Agisoft Metashape. Processamento de um conjunto de imagens de um mesmo voo para a obtenção das nuvens densas de pontos e do MDT. Alinhar Fotos e Construir Nuvem Densa são funções que utilizam-se respectivamente dos algoritmos SfM (Structure from Motion) para gerar a nuvem esparsa e MVS

(Multi-View Stereo) para gerar a nuvem densa

Nesta pesquisa utilizou-se pontos de controle apenas para processamento dos dados obtidos a $100 \mathrm{~m}$ de altura (10 pontos de controle, distribuídos em locais centrais e extremos da área de estudo, levantados com GNSS de dupla frequência portadoras L1/L2 e pós-processados) sendo os outros processos realizados de forma semicontrolada (somente com a informação de localização fornecida pela RPA). Em todos os processos, a opção que determina a quantidade de pontos correspondentes entre as sobreposições das fotos no plano de voo foram de no máximo 40.000 e de no mínimo 4.000 pontos.

Desta forma, obteve-se 9 nuvens de pontos esparsas. Estas foram submetidas ao algoritmo MVS por meio da implementação Build Dense Cloud (Construir Nuvem Densa) com o nível de qualidade Ultra high (Ultra Alta). Com a nuvem de pontos resultante do processamento controlado criou-se uma superfície de referência MDT. A Figura 1 exemplifica duas nuvens densas de pontos semicontroladas (a e b) e o modelo de referência (c) elaborado a partir de uma nuvem de pontos controlada.
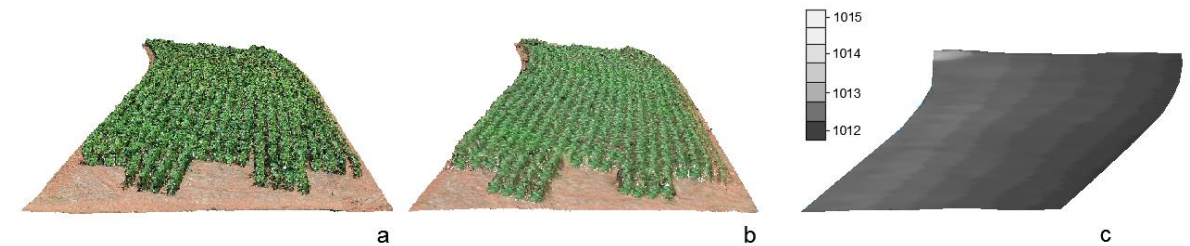

Figura 2. Nuvens de pontos e Modelo Digital de Terreno - MDT. Produtos gerados por processamento SfM - MVS em modo Highest a partir de imagens levantadas a 60 metros de altura (a), 200 metros de altura (b) e 100 metros de altura (c). a e b referem-se as nuvens de pontos e $\mathbf{c}$ ao MDT utilizado

As demais nuvens de pontos (total de 8: resultantes dos processamentos em modo Highest, High, Medium e Low para as alturas de 60 e 200 metros de altura) foram selecionadas por meio de um polígono e exportadas em separado para formato texto, sendo usadas para análise dos diferentes processos de alinhamento no programa CloudCompare versão 2.10.2 (Girardeau-Montaut, 2015).

\section{METODO PARA ESTIMATIVA DA ALTURA}

Inicialmente, as nuvens de pontos referentes as alturas de voo de 60 e 200 metros obtidas em modo Highest e sem a utilização de pontos de controle, foram submetidas ao processo de translação nos eixos XYZ por meio da ferramenta Translate/Rotate, tendo como referência o MDT. A aplicação da técnica é manual e interativa. Visualiza-se a nuvem em diferentes ângulos e aplica-se uma translação em um determinado eixo XYZ, até 
que esta esteja posicionada sobre o MDT, próxima a suas coordenadas reais. Quando isto ocorre, percebe-se visualmente que não é mais necessário mover a nuvem. Apresentam-se informações detalhadas sobre a ferramenta por Girardeau-Montaut (2020).

A translação é necessária devido a baixa acurácia posicional demonstrada pelas nuvens de pontos semicontroladas em relação ao MDT controlado. Após realizar-se as translações, pode-se aplicar a técnica Iterative Closest Point (Iteração com o ponto mais próximo), para um ajuste posicional fino da nuvem. Assim, com a nuvem agora controlada, a altura das plantas de milho pode ser estimada. Seleciona-se o MDT e a nuvem de pontos por meio da interface do programa e em seguida a ferramenta Compute cloud/mesh distance. As distâncias entre o MDT e a nuvem que representam a altura do dossel são então computadas. A figura 3 apresenta o diagrama para obter-se a altura estimada das plantas.

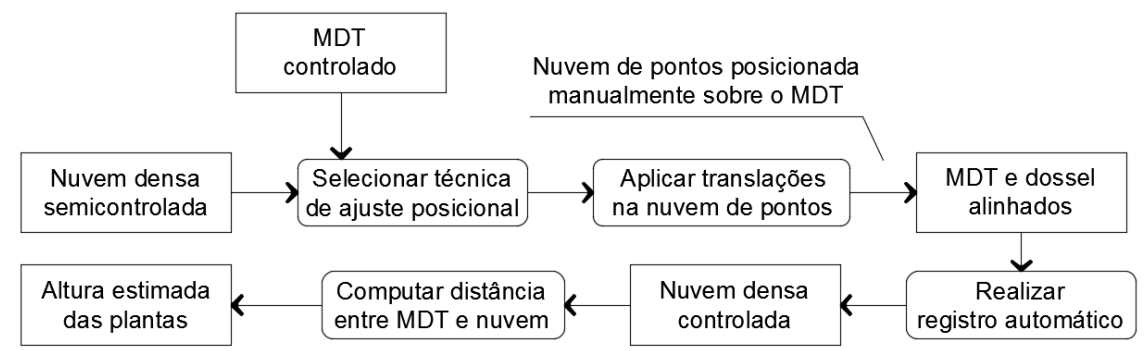

Figura 3. Fluxo de processamento para estimativa da altura das plantas. Existem outras técnicas e parâmetros que podem ser utilizados para ajuste posicional da nuvem como rotação e escala. Propõem-se que sejam executadas somente translações. Não exclui-se a aplicação de filtragem na nuvem, caso esta apresente ruído em excesso, objetivando-se atenuar os efeitos do Fenômeno de Hughes. O registro automático pode não ser necessário para executar a ferramenta Compute cloud/mesh distance pela qual será estimada a altura das plantas

Adicionalmente, analisou-se comparativamente as nuvens de pontos obtidas com um menor custo computacional (High, Medium e Low) com a nuvem obtida pelo processamento Highest, utilizando-se da técnica Compute cloud/cloud distance. Buscou-se com esta análise verificar se seria adequado realizar a metodologia proposta em nuvens obtidas a partir de um menor nível de processamento.

Para utilizar as ferramentas Compute cloud/cloud distance ou Compute cloud/mesh distance seleciona-se os dois arquivos e em seguida uma destas ferramentas. Para executar o processamento basta clicar em computar. Assim, para cada ponto na nuvem ou para cada ponto da malha, o programa irá calcular a distância euclidiana entre os dois arquivos a partir do ponto mais próximo, considerando-se o arquivo de referência.

\section{RESULTADOS}

Demonstra-se pela figura 4 o antes e o depois de aplicar-se a técnica de translação e pela figura 5 os resultados dos cálculos em forma gráfica.

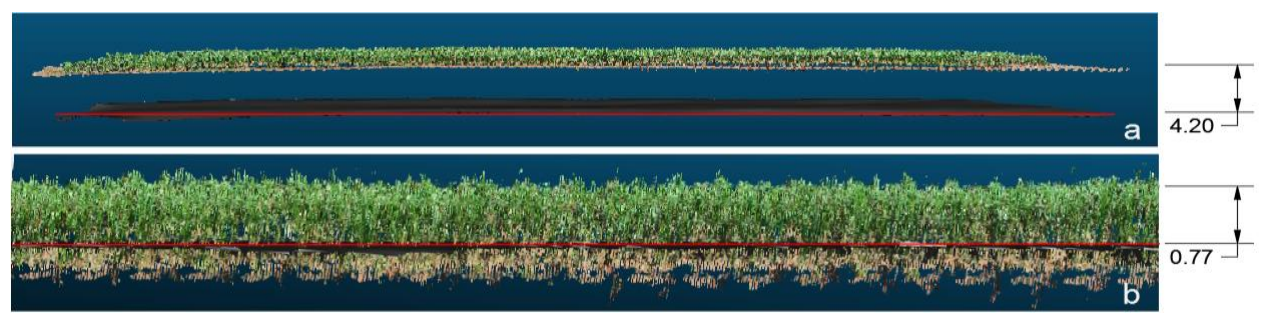

Figura 4. Nuvem de pontos e MDT. As linhas em vermelho demonstram a posição do MDT. Em a demonstra-se a ausência de acurácia posicional da nuvem de pontos, na distância média de 4,20 m da superfície do terreno. Em b demonstra-se em detalhe a altura aproximada das plantas de uma parte da parcela $(0,77 \mathrm{~m})$, após a aplicação da translação da nuvem de pontos. Infere-se a a altura das plantas pelo cálculo da distância euclidiana entre o MDT e os pontos da nuvem em verde pelos quais representa-se a lavoura de milho, utilizando-se técnica Compute cloud/mesh distance 

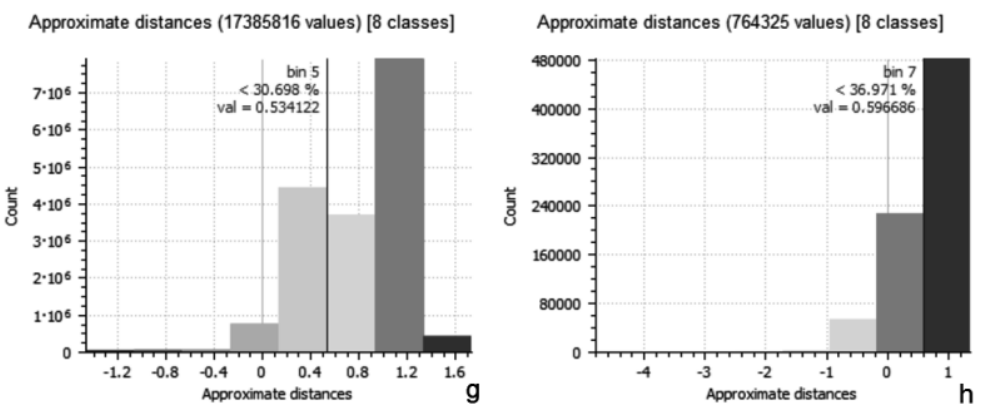

Figura 5. Faixa de altura predominante das plantas de milho. Resultados das análises em forma gráfica dos cálculos da distância euclidiana em metros entre o modelo MDT criado utilizando-se de pontos de controle e as nuvens de pontos resultantes do processamento em modo Highest sem a utilização de pontos de controle, após a aplicação da técnica de translação nos eixos XYZ (gráfico g para a altura de voo de $60 \mathrm{~m}$ e gráfico $\mathbf{h}$ para a altura de voo de $200 \mathrm{~m}$ ). A faixa de altura predominante foi de $66,87 \%$ das plantas entre 0,53 - 1,33 metros (voo a $60 \mathrm{~m}$ do solo) e $63,02 \%$ das plantas entre 0,59 - 1,36 metros (voo a $200 \mathrm{~m}$ do solo)

Com a análise comparativa das nuvens de pontos, demonstra-se as diferenças entre as nuvens obtidas com menor custo computacional (High, Medium e Low) e a nuvem com maior nível de processamento (parâmetro Accuracy em modo Highest). Os resultados dos cálculos são apresentados em forma gráfica na figura 6 e organizados na tabela 1 .

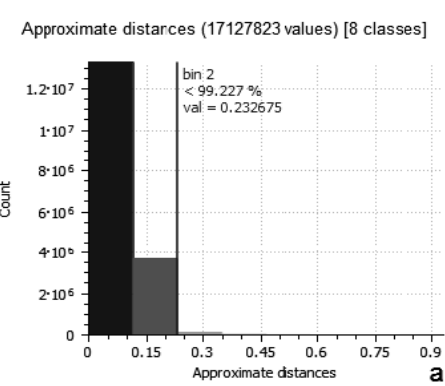

Approximate distences (777757 values) [8 classes]

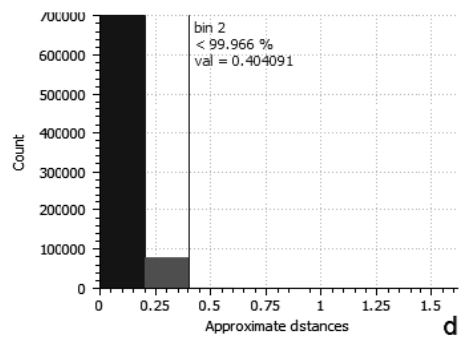

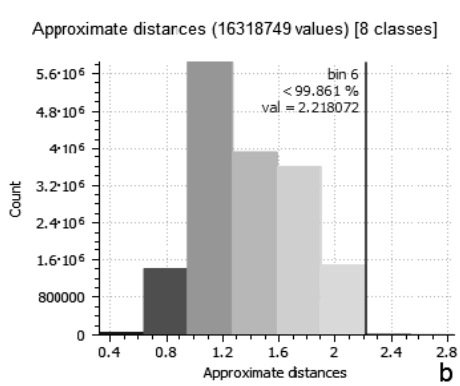

Approximate distances (815529 values) [8 classes]

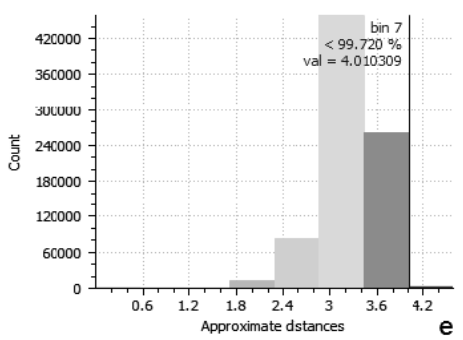

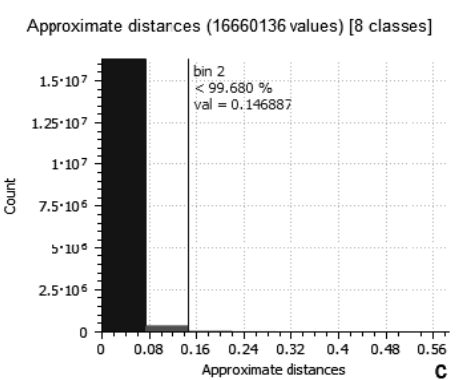

Approximate distences (727907 values) [8 classes]

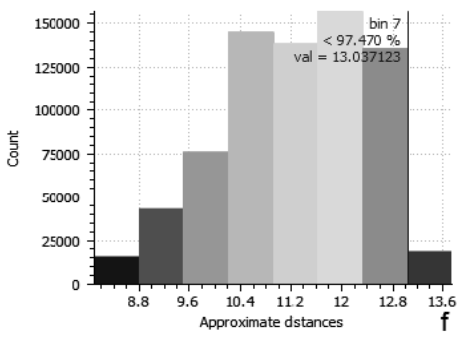

Figura 6. Resultados das comparações entre as diferentes nuvens de pontos. Resultados das análises em forma gráfica dos cálculos das distâncias euclidianas em metros entre as nuvens de pontos resultantes de diferentes processos de alinhamento fotogramétrico das imagens RPA obtidas em diferentes alturas de voo na data de 03/12/2019 (a, b e c referem-se à altura de voo de $60 \mathrm{~m}$ e d, e e f a altura de $200 \mathrm{~m}$ de voo) 
Tabela 1. Distâncias obtidas entre as nuvens de pontos conforme às alturas de voo. Dados determinados a partir dos gráficos obtidos. Demonstra-se que não ocorreram diferenças significativas entre os pontos tridimensionais, quando comparados os processos de alinhamento Highest e High (gráficos a e d). Porém ocorreram altas variações e grande aumento das distâncias tridimensionais entre os pontos quando realizou-se as outras comparações: Highest e Medium (0,95 - 2,21 metros no gráfico b e 2,86 - 4,01 metros no gráfico e) e Medium / Low para os processamentos das imagens obtidas a $200 \mathrm{~m}$ de altura $(9,50$ - 13,03 metros no gráfico f)

\begin{tabular}{lcccccc}
\hline Altura de voo & \multicolumn{6}{c}{ Distância mínima e máxima entre duas nuvens de pontos em metros } \\
\hline \multirow{4}{*}{ metros } & Highest / High & $\%$ & Highest / Medium & $\%$ & Medium / Low & $\%$ \\
200 metros & $0-0,22$ & 99,22 & $0,95-2,21$ & 90,98 & $0-0,07$ & 97,67 \\
& $0-0,44$ & 99,96 & $2,86-4,01$ & 88,14 & $9,50-13,03$ & 89,45 \\
\hline
\end{tabular}

\section{CONCLUSÕES}

Demonstra-se pelos resultados que a diferença entre as nuvens de pontos levando-se em consideração o nível de processamento de maior custo computacional como referência (Highest) é diretamente proporcional a altura de voo. Outra conclusão é a de que independente da altura de voo, as diferenças entre os processamentos nos modos Highest e High não são significativas quando considerado um processamento semicontrolado. Assim conclui-se que, mediante a aplicação da técnica em uma grande quantidade de dados, que levaria um maior tempo de processamento, pode-se optar pelo parâmetro High, pois a distância computacional entre os pontos das nuvens não se mostraram significativas.

Em relação a capacidade de estimar a altura das plantas de milho por meio do método apresentado, foi possível determinar a faixa de altura predominante das plantas independente da altura de voo. Ou seja, demonstra-se que a menor quantidade de pontos utilizados para medição da nuvem (referente ao voo realizado a $200 \mathrm{~m}$ de altura), não influenciou significativamente os resultados. Desta forma, também pode-se inferir que um menor nível de processamento da nuvem que gere menos pontos e com menor custo computacional, não interfira na estimativa da altura das plantas. Porém, necessita-se realizar mais testes de medição das distancias entre o MDT e nuvens obtidas pelos parâmetros de High, Medium e Low.

Apesar das alturas não terem sido medidas em solo na data dos voos, a média gerada de $0,78 \mathrm{~m}$ no computo das distâncias é coerente com o estádio fenológico em que encontrava-se o milho (V8-V9) e com a estatura indicada para aplicação preventiva de fungicida (em torno de 0,80 m), conforme realizou-se 3 dias antes dos levantamentos em 29/11/2019.

Apresenta-se por meio deste trabalho, resultados parciais sobre um projeto de monitoramento de parâmetros biofísicos da cultura do milho. Outros voos foram realizados e estão sendo analisados.

\section{AGRADECIMENTOS}

A Fundação Araucária e ao Serviço Nacional de Aprendizagem Rural (SENAR), pela bolsa de estudo.

\section{REFERÊNCIAS}

Agisoft, 2017. Agisoft PhotoScan User Manual: Professional Edition, Version 1.3.0. Acessado em 18 de junho de 2020. http://www.agisoft.com/pdf/photoscan_python_api_1_3_0.pdf

Bending, J. et al., 2014. Estimating biomass of barley using crop surface models (CSMs) derived from UAV-based RGB imaging. Remote Sensing, Vol. 6, No. 11, 10395 - 10412.

Enciso, J. et al., 2019. Validation of agronomic UAV and field measurements for tomato varieties. Computers and Electronics in Agriculture, Vol. 158, p. 278-283.

Girardeau-Montaut, D., 2015. CloudCompare Version 2.6.1 User Manual.

Girardeau-Montaut, D., 2020. Translate along and rotate around any arbitrary vector. Acessado em 25 de agosto de 2020. https://github.com/CloudCompare/CloudCompare/pull/1031

Grenzdörffer, G. J., 2014. Crop height determination with UAS point clouds. ISPRS Archives, Denver, Colorado, USA, Vol. XL-1, p. 135-140. 